\title{
Resistance to cefotaxime and peptidoglycan composition in Enterococcus faecalis are influenced by exogenous sodium chloride
}

\author{
Jean-Luc Mainardi, ${ }^{1,2}$ Danièle Billot-Klein, ${ }^{2}$ Anne Coutrot, ${ }^{1}$ \\ Raymond Legrand, ${ }^{3}$ Bernard Schoot ${ }^{3}$ and Laurent Gutmann ${ }^{2}$
}

Author for correspondence: Jean-Luc Mainardi. Tel: +331423468 63. Fax: +33143256812.

1 Hôpital Saint-Joseph, Service de Microbiologie Clinique, 185 rue Raymond Losserand, 75674 Paris Cedex 14, France

2 LRMA, Université Paris VI, 15 , rue de l'Ecole de Médecine, 75270 Paris Cedex 06, France

3 Physics Department, Roussel UCLAF, 93230 Romainville, France

\begin{abstract}
The influence of $\mathrm{NaCl}$ on the susceptibility of Enterococcus faecalis to cefotaxime was tested with JH2-2, a laboratory strain, and 20 clinical strains grown on tryptic soy agar supplemented with $5 \%$ horse blood. Growth with $3 \% \mathrm{NaCl}$ in the medium resulted in an increase in cefotaxime resistance and the appearance of a heterogeneous resistance phenotype: for the majority of the strains, the MICs of cefotaxime increased from 4 to $512 \mu \mathrm{g} \mathrm{ml}^{-1}$. By a competition assay using cefotaxime and $\left[{ }^{3} \mathrm{H}\right]$ benzylpenicillin, it was shown for strain JH2-2 that at the MIC penicillin-binding protein (PBP) 2 and PBP3 were the apparent essential PBPs in medium without $\mathrm{NaCl}$, whilst the low-affinity PBPs 4 and 1 were the apparent essential PBPs for cell growth in medium containing $3 \% \mathrm{NaCl}$. Analysis of JH2-2 peptidoglycan by HPLC and MS after growth in the presence of $3 \% \mathrm{NaCl}$ showed a relative increase in unsubstituted monomers and a relative decrease in alanine- and dialanine-substituted monomers. It is therefore hypothesized that modification of the number of alanine-substituted precursors in the presence of $\mathrm{NaCl}$ could interfere with the functions of the different PBPs and thus play a role in cefotaxime resistance in E. faecalis.
\end{abstract}

Keywords : cefotaxime resistance, sodium chloride, Enterococcus faecalis, peptidoglycan, penicillin-binding proteins

\section{INTRODUCTION}

Enterococcus faecalis is a bacterium isolated with increasing frequency from a wide variety of infections, including nosocomial infections (Jarvis \& Martone, 1992; Vincent et al., 1995). Enteroccoci are considered naturally resistant to cefotaxime, a third-generation cephalosporin, as well as to other cephalosporins. However, as shown in this study, depending on the medium, E. faecalis can appear susceptible to cefotaxime in vitro. This is especially marked in tryptic soy agar supplemented with $5 \%$ horse blood. Moreover, in Enterococcus hirae ATCC 9790, susceptibility to $\beta$ lactam antibiotics, particularly cefotaxime, has been shown to be influenced by different growth conditions, especially temperature (Fontana et al., 1983; Canepari et al., 1986, 1987). An important feature of certain methicillin-resistant Staphylococcus aureus strains is that they can segregate a small proportion of highly resistant subclones, a phenomenon referred to as heterogeneous resistance. The expression of this heterogeneous $\beta$-lactam resistance has been shown to be influenced by different external factors such as the concentration of $\mathrm{NaCl}$ (Sabbath, 1977). Therefore, by analogy with these phenomena, we investigated the influence of $\mathrm{NaCl}$ on the susceptibility of E. faecalis to cefotaxime and on the composition of its peptidoglycan.

\section{METHODS}

Bacterial strains. Twenty recently isolated clinical isolates of E. faecalis and $\mathrm{JH} 2-2$, a reference strain (Jacob \& Hobbs, 1974), were studied. The bacteria were identified by usual procedures (Facklam \& Collins, 1989). Strains were cultivated without aeration at $37^{\circ} \mathrm{C}$ in brain heart infusion medium (Difco).

Antibiotics. Cefotaxime was provided by Roussel UCLAF. $\left[{ }^{3} \mathrm{H}\right]$ Benzylpenicillin $\left(0.66 \mathrm{TBq} \mathrm{mmol}^{-1}\right)$ was synthesized at 


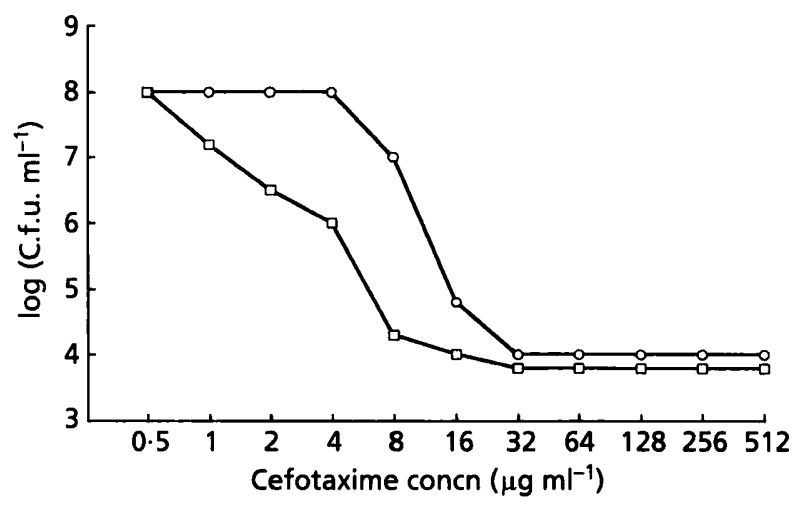

Fig. 1. Population analysis profiles of $\mathrm{JH2}-2$. Cells were grown in tryptic soy broth and plated at several dilutions on TSA with $5 \%$ horse blood with (O) or without $(\square) 3 \% \mathrm{NaCl}$ and containing various concentrations of cefotaxime. After $18 \mathrm{~h}$ at $37^{\circ} \mathrm{C}$, numbers of colonies were counted and expressed as log (c.f.u. $\mathrm{ml}^{-1}$ ).

the Service des Molécules Marquées, Commissariat à l'Energie Atomique (Gif-sur-Yvette, France) and was generously provided by Rhône-Poulenc Recherche (Vitry-sur-Seine, France).

MICs and resistance phenotype. MICs were determined on tryptic soy agar (TSA; Difco) supplemented with $5 \%$ horse blood containing serially twofold-diluted cefotaxime, with or without $3 \% \mathrm{NaCl}$. Plates were inoculated with a Steers-type device $\left(10^{4}\right.$ c.f.u. per spot) and incubated at $37^{\circ} \mathrm{C}$ for $18 \mathrm{~h}$.

Phenotypic expression of resistance was determined by population analysis profiles on TSA plates supplemented with $5 \%$ horse blood, with or without $3 \% \mathrm{NaCl}$, and containing various concentrations of cefotaxime $\left(0 \cdot 5-512 \mu \mathrm{g} \mathrm{ml}^{-1}\right)$. After $18 \mathrm{~h}$ at $37^{\circ} \mathrm{C}$, numbers of colonies were counted and expressed as $\log$ (c.f.u. $\mathrm{ml}^{-1}$ ).

Analysis of penicillin-binding proteins (PBPs) of JH2-2. PBP analysis was performed with a competition assay as previously described (Mainardi et al., 1995), except that bacteria were directly resuspended in sodium phosphate buffer $(50 \mathrm{mM}$, $\mathrm{pH} 7$ ) after growth for 12-14 h on TSA supplemented with $5 \%$ horse blood, with or without $3 \% \mathrm{NaCl}$. The $\mathrm{OD}_{650}$ was $0 \cdot 5$. The concentrations of non-radioactive cefotaxime varied from 4 to $512 \mu \mathrm{g} \mathrm{ml}^{-1}$ and that of $\left[{ }^{3} \mathrm{H}\right]$ benzylpenicillin was $20 \mu \mathrm{g} \mathrm{ml}^{-1}$. Exposure times for the fluorograms ranged from 10 to $30 \mathrm{~d}$. The densities of the bands were determined by scanning densitometry (Personnal Densitometer SI, Molecular Dynamics).

Muropeptide composition of JH2-2 peptidoglycan. The analysis of peptidoglycan was performed by reverse-phase HPLC and by MS as previously described (Billot-Klein et al., 1997), except that bacteria were directly resuspended in sodium phosphate buffer $(50 \mathrm{mM}, \mathrm{pH} 7)$ after growth for 12-14 h on TSA supplemented with $5 \%$ horse blood, with or without $3 \% \mathrm{NaCl}$. The structure of the peaks was deduced from their molecular masses and according to the peptidoglycan composition of $\mathrm{JH} 2-2$ as described by de Jonge et al. (1996).

\section{RESULTS}

\section{MICs and resistance phenotype}

For 16 out of 20 clinical strains and for JH2-2, the MIC of cefotaxime increased from a mean of 4 (range 2-8) $\mu \mathrm{g}$ $\mathrm{ml}^{-1}$ to a mean of 512 (range 128-1024) $\mu \mathrm{g} \mathrm{ml}^{-1}$ in the presence of $3 \% \mathrm{NaCl}$. For the other four strains, the MIC increased from 64 to $512 \mu \mathrm{g} \mathrm{ml}^{-1}$. Conversely, the presence of $\mathrm{NaCl}$ did not modify the susceptibility to penicillin and ampicillin (data not shown). Fig. 1 shows population analysis profiles of $\mathrm{JH} 2-2$ grown with or without $3 \% \mathrm{NaCl}$ in the presence of various concentrations of cefotaxime. Increased concentrations of cefotaxime caused heterogeneous expression of resistance with an increase in cefotaxime resistance for the majority of the cells in the presence of $\mathrm{NaCl}$. Colonies picked from a plate containing $3 \% \mathrm{NaCl}$ and $64 \mu \mathrm{g}$ cefotaxime $\mathrm{ml}^{-1}$ appeared susceptible to cefotaxime $\left(2 \mu \mathrm{g} \mathrm{ml}^{-1}\right)$ when replated on TSA with $5 \%$ horse blood without $\mathrm{NaCl}$, but expressed heterogeneous resistance when plated on TSA with $5 \%$ horse blood and $3 \%$ $\mathrm{NaCl}$ (data not shown). Conversely, in the absence of (a)

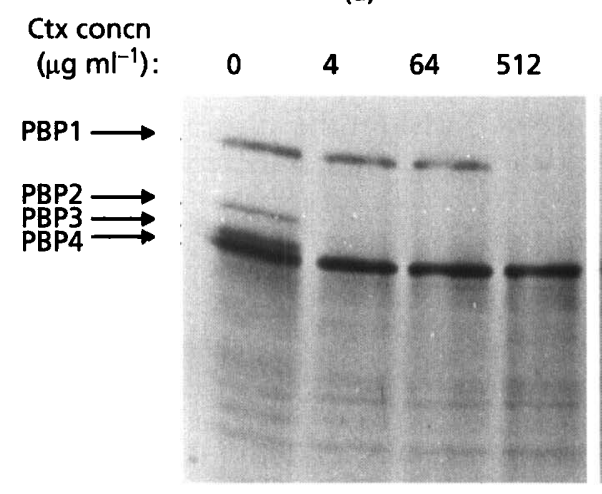

(b)

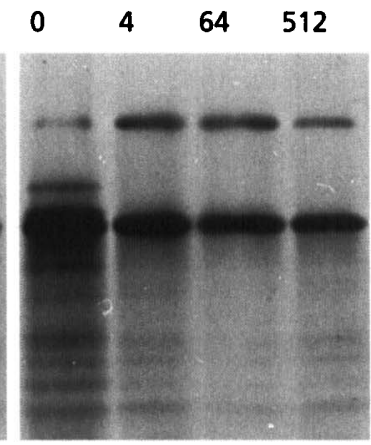

(c)

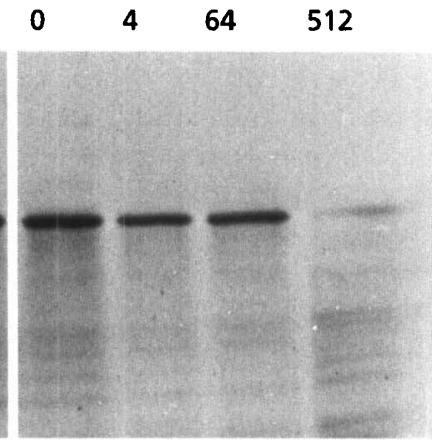

Fig. 2. Saturation of PBPs of $\mathrm{JH} 2-2$ by cefotaxime ( $\mathrm{Ctx}$ ) grown without (a) or with $3 \% \mathrm{NaCl}(\mathrm{b})$. (c) Saturation of PBPs of a cefotaxime-resistant mutant of $\mathrm{JH}_{2}-2$ by cefotaxime. Competition experiments with $\left.{ }^{3} \mathrm{H}\right]$ benzylpenicillin $\left(20 \mu \mathrm{g} \mathrm{ml} \mathrm{m}^{-1}\right)$ were carried out after addition of increasing concentrations of cefotaxime. 


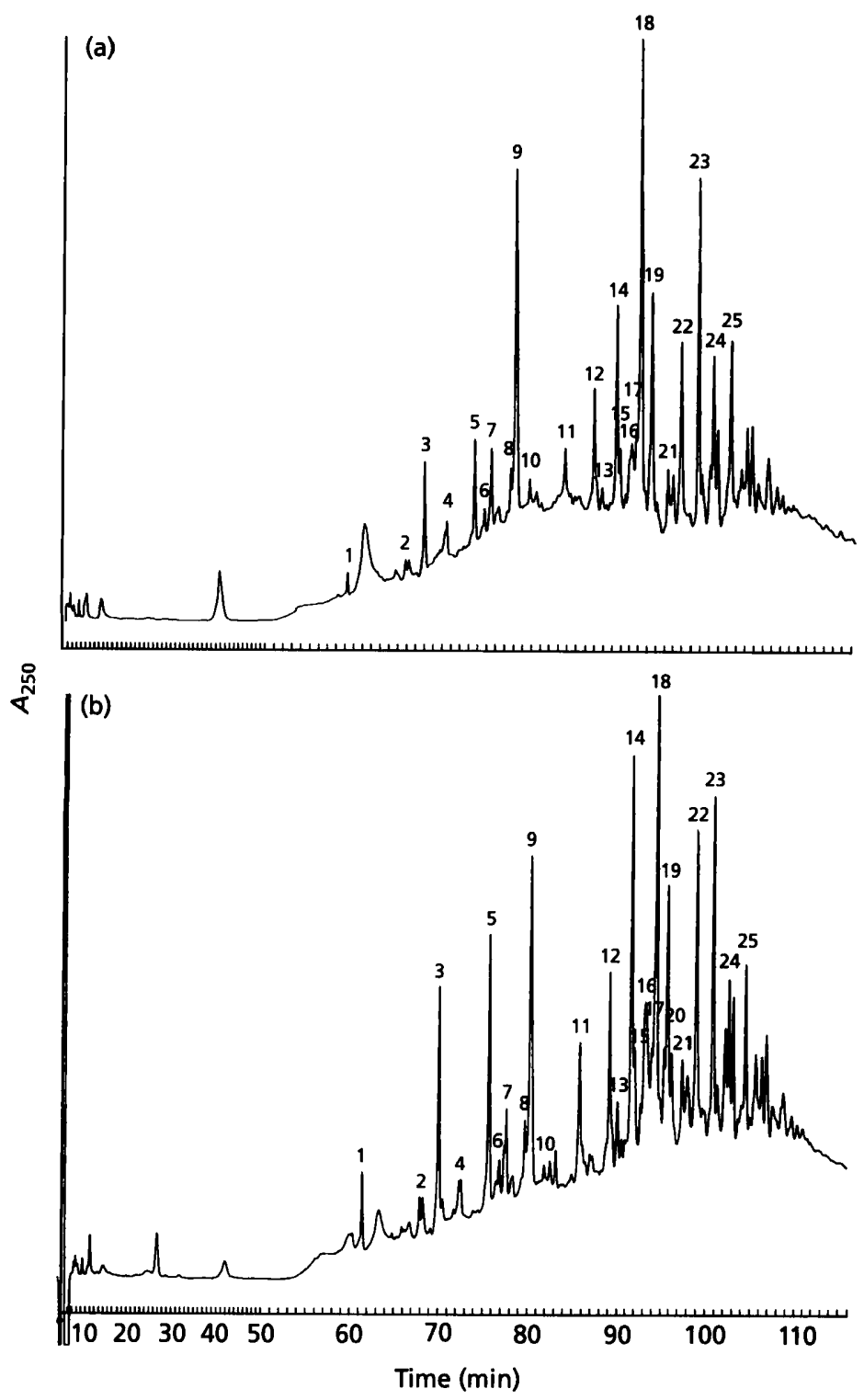

Fig. 3. Separation of E. faecalis $\mathrm{JH} 2-2$ cell wall muropeptides by reverse-phase HPLC. Peptidoglycan was obtained after growth without (a) or with (b) $3 \% \mathrm{NaCl}$. After concentration by centrifugation, the cells were boiled with $4 \%$ SDS. Cell walls were then purified by using Pronase followed by trypsin. Peptidoglycan was hydrolysed with lysozyme and mutanolysin. Separation of the digested cell-wall components was performed on an HPLC system. The column was eluted at a flow rate of $0.5 \mathrm{ml} \mathrm{min}^{-1}$ for $10 \mathrm{~min}$ with $0.05 \%(\mathrm{v} / \mathrm{v})$ trifluoroacetic acid in water and subsequently with a $90 \mathrm{~min}$ linear acetonitrile gradient $(0-20 \%)$ in $0.035 \%$ trifluoroacetic acid. Peak material was characterized by liquid chromatography-MS. Numbers correspond to the peaks identified in Table 1.
$\mathrm{NaCl}$ and at concentrations of cefotaxime of $\geqslant 16 \mu \mathrm{g}$ $\mathrm{ml}^{-1}$, a population of cefotaxime-resistant mutants, which differed from the population obtained in the presence of $\mathrm{NaCl}$, was selected at a high frequency of $10^{-4}$ (Fig. 1). These mutants expressed homogeneous resistance to cefotaxime and are referred to as cefotaxime-resistant mutants (MIC $\geqslant 512 \mu \mathrm{g} \mathrm{ml}^{-1}$ ).

\section{PBPs of JH2-2}

After growth in the absence of $\mathrm{NaCl}$, and at a concentration of cefotaxime $\left(4 \mu \mathrm{g} \mathrm{ml}^{-1}\right)$ equivalent to the MIC, the competition assay showed that PBPs 2 and 3 were fully saturated (Fig. 2a), suggesting that under these conditions these are the essential PBPs (Mainardi et al., 1995). In the presence of $\mathrm{NaCl}$, scanning densitometry showed an increase in the amount of all PBPs but particularly PBPs 4 and 1 ; the ratio of the quantity of $\mathrm{PBP}$ in the presence of $\mathrm{NaCl}$ to the quantity of PBP in the absence of $\mathrm{NaCl}$ was 1.74 for PBP4, 1.8 for PBP1, $1 \cdot 17$ for PBP2 and 1.10 for PBP3. Again, PBPs 2 and 3 were saturated with low concentrations of cefotaxime. At a cefotaxime concentration equivalent to the MIC (512 $\mu \mathrm{g}$ $\left.\mathrm{ml}^{-1}\right)$, PBP1 was only partially acylated $(\sim 70 \%)$, and PBP4 was very poorly acylated $(<15 \%$ ) (Fig. 2b) indicating that, in the presence of $\mathrm{NaCl}$, either PBP1 or PBP4, or both, were able to take over the functions of PBPs 2 and 3. In contrast to these findings, the cefotaxime-resistant mutants selected in the absence of $\mathrm{NaCl}$ barely expressed PBPs 1 and 2, lacked PBP3 and expressed preferentially only PBP4 (Fig. 2c).

\section{Muropeptide composition of JH2-2 peptidoglycan}

To determine the effect of $\mathrm{NaCl}$ on the peptidoglycan composition of $\mathrm{JH} 2-2$ grown in the absence or presence of $\mathrm{NaCl}$, its structure was studied by HPLC and mass spectrometry (Fig. 3, Table 1). The relative amounts of 
Table 1. Molecular mass and composition of muropeptides from E. faecalis JH2-2 peptidoglycan

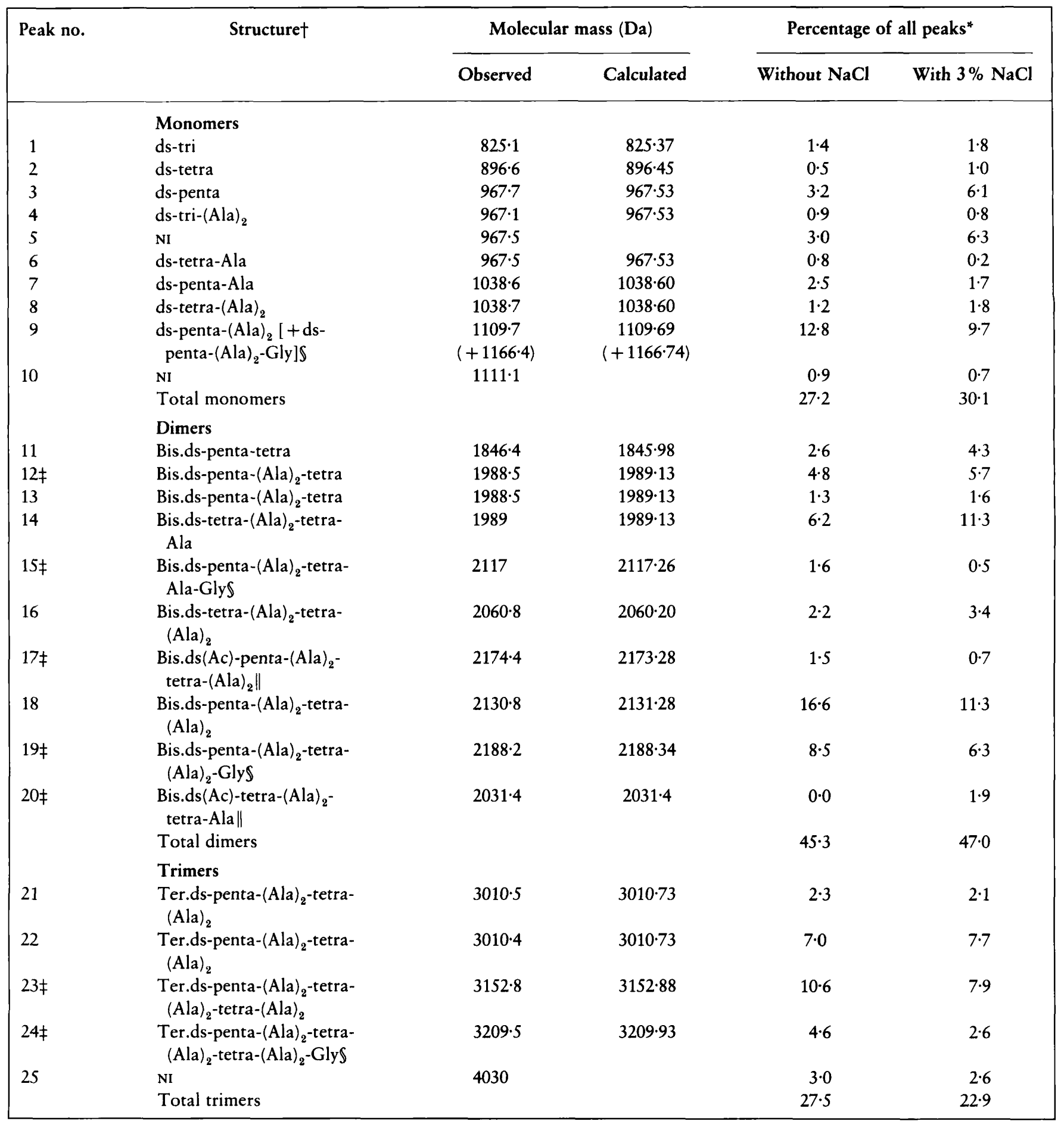

*Values shown are percentages of the sum of all of the integrated peak areas presented in the table.

†ds, disaccharide (GlcNAc-MurNAc); Bis, dimeric form; Ter, trimeric form; tri, tripeptide (L-Ala-D-iGln-L-Lys); tetra, tetrapeptide (L-Ala-D-iGln-L-Lys-D-Ala); penta, pentapeptide (L-Ala-D-iGln-L-Lys-D-Ala-D-Ala); NI, precise structure has not been identified. $\ddagger$ Peaks not previously identified by de Jonge et al. (1996); structures deduced from their molecular masses and retention time. $\$$ Molecular mass difference with neighbouring peak of $57 \mathrm{Da}$ suggests the presence of a glycine.

\| Excess molecular mass of $42 \mathrm{Da}$ suggests the $\mathrm{O}$-acetylation of one MurNAc residue. 
Table 2. Percentage of identified unsubstituted or substituted monomers

$100 \%$ represents monomers of which structures have been identified.

\begin{tabular}{|lcc|}
\hline Monomer & Without $\mathbf{N a C l}$ & With NaCl \\
\hline Unsubstituted monomers in peaks 1, 2 and 3 & 15 & 37 \\
Alanine-substituted monomers in peaks 6 and 7 & 19 & 8.7 \\
Dialanine-substituted monomers in peaks 4, 8 and 9 & 66 & $54 \cdot 3$ \\
\hline
\end{tabular}

Table 3. Percentage of identified unsubstituted and substituted dissacharide pentapeptide monomers (ds-penta)

$100 \%$ represents all identified ds-pentas.

\begin{tabular}{|lcc|}
\hline ds-penta & Without NaCl & With NaCl \\
\hline $\begin{array}{l}\text { Unsubstituted ds-penta in } \\
\text { peak 3 }\end{array}$ & $17 \cdot 3$ & $33 \cdot 7$ \\
$\begin{array}{c}\text { Alanine substituted } \\
\text { ds-penta in peak 7 } \\
\text { Dialanine substituted } \\
\text { ds-penta in peak 9 }\end{array}$ & 13.6 & $9 \cdot 8$ \\
\hline
\end{tabular}

the different muropeptides are shown in Table 1. Although only a few changes in the overall proportions of monomers, dimers and trimers were noted, differences in the relative quantities of muropeptides, particularly among the monomers, were found when $\mathrm{JH} 2-2$ was grown with $\mathrm{NaCl}$. When all monomers were considered alone, the comparison between the quantities of identified unsubstituted and substituted disaccharide-peptides showed that in the presence of $\mathrm{NaCl}$ there was a relative increase in unsubstituted disaccharide-peptides and a decrease in the substituted disaccharide-peptides (Tables $1,2)$. This was also the case when the monomer pentapeptides were considered alone (Table 3 ).

When relative quantities of the major dimeric structures were considered (Table 1, Fig. 3, peaks 11, 12, 14, 18, 19), the quantity of the pentatetrapeptide without alanine substitution (peak 11) was increased in the presence of $\mathrm{NaCl}$; indeed, with respect to the total dimeric structures, peak 11 represented $8.9 \%$ when strains were grown in the presence of $\mathrm{NaCl}$ and $6.1 \%$ in the absence of $\mathrm{NaCl}$. Similarly, the quantities of dimeric stuctures with only two or three alanine substitutions (peaks 12 and 14) were increased $(+1.2$ and $+9.4 \%$, respectively), whilst those containing four alanines (peaks 18 and 19) were decreased $(-13.8$ and $-6 \%$, respectively).

Interestingly, when the peptidoglycan composition of a cefotaxime-resistant mutant which expressed exclusively PBP4 was examined after growth in the absence of $\mathrm{NaCl}$, neither quantitative nor qualitative changes were observed compared to JH 2-2 grown in the absence of $\mathrm{NaCl}$ (data not shown).

\section{DISCUSSION}

For E. hirae (ATCC 9790) changes in growth temperature and medium composition were found to cause striking changes in penicillin susceptibility, in peptidoglycan synthesis in the presence of penicillin, and in the labelling pattern of PBPs (Fontana et al., 1983). This suggested that different PBPs were involved in peptidoglycan synthesis depending on the growth conditions. In another study (del mar Lleò et al., 1987), the susceptibility of $E$. hirae to cefotaxime was shown to be influenced also by the growth temperature and related to the saturation of different PBPs.

In our study of $E$. faecalis, the addition of $\mathrm{NaCl}$ to the growth medium led to an increase in cefotaxime resistance and yielded a heterogeneous phenotype. The PBP pattern of $E$. faecalis $\mathrm{JH} 2-2$ was similar in the presence or absence of $\mathrm{NaCl}$, although a relative increase in amounts of PBPs 1 and 4 was observed in the presence of $\mathrm{NaCl}$.

The increase in resistance in the presence of $\mathrm{NaCl}$, without important modifications in the PBP patterns, was associated with quantitative alterations of the muropeptide. The main peptidoglycan modification after growth in the presence of $\mathrm{NaCl}$ was a decrease in alanine-substituted monomers. We therefore hypothesize that, depending on the presence or the absence of $\mathrm{NaCl}$, the quantitative variations of the alanine-substituted monomers in the muropeptide, and therefore in the peptidoglycan precursors, could require the function of different PBPs, explaining, in turn, the MIC variations observed.

Taking into account the different results, we tentatively built a speculative model. In the absence of $\mathrm{NaCl}$ (Fig. 4a), predominantly substituted precursors would be synthesized and preferentially processed by PBPs 2 and 3. Therefore, these PBPs would become essential and their saturation at $4 \mu \mathrm{g} \mathrm{ml}^{-1}$ would explain the low MIC of cefotaxime. Conversely, in the presence of $\mathrm{NaCl}$ (Fig. 4b), predominantly unsubstituted precursors would be synthesized, which would be preferentially processed by PBPs 4 and 1 . Since these PBPs are not fully acylated at $512 \mu \mathrm{g} \mathrm{ml}^{-1}$, they would become the essential PBPs and explain the high MICs observed for cefotaxime in the presence of $\mathrm{NaCl}$.

As far as the 'truly' cefotaxime-resistant mutants are concerned, in which PBP4 is almost exclusively expressed, the low affinity of this PBP would explain the 


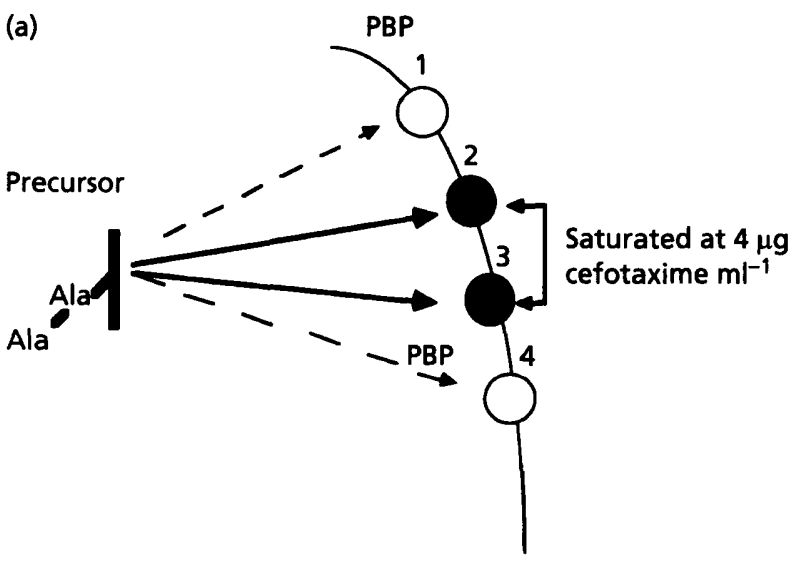

(b)

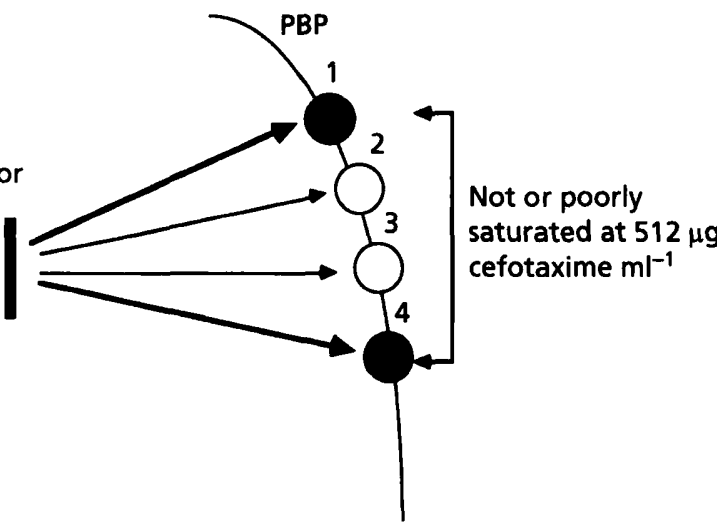

Fig. 4. Proposed speculative model for the processing of precursors by different PBPs under different growth conditions. (a) In the absence of $\mathrm{NaCl}$ the predominantly substituted precursors are preferentially processed by PBPs 2 and 3; (b) in the presence of $3 \% \mathrm{NaCl}$ the predominantly unsubstituted precursors are preferentially processed by PBPs 1 and 4.

high level of resistance observed. Since no $\mathrm{NaCl}$ was necessary to express the resistance of these mutants, it is not expected that changes in the peptidoglycan had occurred provided that the now essential PBP4 could process sufficient amounts of the predominantly substituted precursors in the absence of the other PBPs.

This study has identified an environmental parameter that affects cefotaxime resistance in E. faecalis. $\mathrm{NaCl}$ could interfere either with expression of genes involved in the synthesis of the alanine side chain characteristic of E. faecalis peptidoglycan, or directly with the different enzymes involved in the addition of alanine in the crossbridge. Although little is known about the addition of alanine to the peptidoglycan precursor, it was suggested that, similarly to Lactobacillus viridescens (Plapp \& Strominger, 1970), the alanine (1 or 2) present in the interpeptide bridge was successively added from the L-alanyl tRNA by different enzymes. The first one would be added to the $\varepsilon$-amino group of the lysine and the second, when present, to the first branched alanine. By analogy, it is interesting to note that in staphylococci under $\mathrm{NaCl}$ stress, shorter interpeptide glycine bridges were observed (Vijaranakul et al., 1995) and that modification of the glycine content of the side chain of cell-wall precursors, after inactivation of different fem genes, explained in part the heterogeneous resistance to methicillin (de Lencastre et al., 1994; Berger-Bächi, 1994).

\section{ACKNOWLEDGEMENTS}

The work was supported by a grant (CRI 950601) from the Institut National de la Santé et de la Recherche Médicale, Paris, France. We thank J. van Heijenoort (Biochimie Moléculaire et Cellulaire, CNRS, Orsay, France) for critically reading the manuscript.

\section{REFERENCES}

Berger-Băchi, B. (1994). Expression of resistance to methicillin. Trends Microbiol 2, 389-392.

Billot-Klein, D., Legrand, R., Schoot, B., van Heijenoort, J. \& Gutmann, L. (1997). Peptidoglycan structure of Lactobacillus casei, a species highly resistant to glycopeptide antibiotics. J Bacteriol 179, 6208-6212.

Canepari, P., del mar Lled, M. M., Cornaglia, G., Fontana, R. \& Satta, G. (1986). In Streptococcus faecium penicillin-binding protein 5 alone is sufficient for growth at sub-maximal but not at maximal rate. J Gen Microbiol 132, 625-631.

Canepari, P., del mar Lled, M. M., Fontana, R. \& Satta, G. (1987). Streptococus faecium mutants that are temperature sensitive for cell growth and show alterations in penicillin-binding proteins. J Bacteriol 169, 2432-2439.

Facklam, R. \& Collins, M. D. (1989). Identification of Enterococcus species isolated from human infections by a conventional test scheme. J Clin Microbiol 27, 731-734.

Fontana, R., Canepari, P., Satta, G. \& Coyette, J. (1983). Streptococcus faecium ATCC 9790 penicillin-binding proteins and penicillin sensitivity are heavily influenced by growth conditions: proposal for an indirect mechanism of growth inhibition by $\beta$-lactams. $J$ Bacteriol $154,916-923$.

Jacob, A. E. \& Hobbs, S. J. (1974). Conjugal transfer of plasmidborne multiple antibiotic resistance in Streptococcus faecalis var. zymogenes. J Bacteriol 117, 360-372.

Jarvis, W. R. \& Martone, W. J. (1992). Predominant pathogens in hospital infections. J Antimicrob Chemother 29, Suppl. A, 19-24.

de Jonge, B. L. M., Handwerger, S. \& Gage, D. (1996). Altered peptidoglycan composition in vancomycin-resistant Enterococcus faecalis. Antimicrob Agents Chemother 40, 863-869.

de Lencastre, H., de Jonge, B. L. M., Matthews, P. R. \& Tomasz, A. (1994). Molecular aspects of methicillin resistance in Staphylococcus aureus. J Antimicrob Chemother 33, 7-24.

del mar Lled, M. M., Canepari, P., Cornaglia, G., Fontana, R. \& Satta, G. (1987). Bacteriostatic and bactericidal activities of $\beta$ lactams against Streptococcus (Enterococcus) faecium are associated with saturation of different penicillin-binding proteins. Antimicrob Agents Chemother 31, 1618-1626.

Mainardi, J. L., Gutmann, L., Acar, J. F. \& Goldstein, F. W. (1995). Synergistic effect of amoxicillin and cefotaxime against Enterococcus faecalis. Antimicrob Agents Chemother 39, $1984-1989$.

Plapp, R. \& Strominger, J. L. (1970). Biosynthesis of peptidoglycan and of interpeptide bridges in Lactobacillus viridescens. J Biol Chem 245, 3667-3674. 
Sabbath, L. D. (1977). Chemical and physical factors influencing methicillin resistance of Staphylococcus aureus and Staphylococcus epidermidis. J Antimicrob Chemother 3, 47-51.

Vijaranakul, U., Nadakavukaren, M. J., de Jonge, B. L. M., Wilkinson, B. J. \& Jayaswal, R.K. (1995). Increased cell size and shortened peptidoglycan interpeptide bridge of $\mathrm{NaCl}$-stressed Staphylococcus aureus and their reversal by glycine betaine. J Bacteriol 177, 5116-5121.
Vincent, J. L., Bihari, D. J, Suter, P. H., Bruining, H. A., White, J. \& Nicolas-Chanoine, M. H. (1995). The prevalence of nosocomial infections in intensive care units in Europe. Results of the European prevalence of infection in intensive care (EPIC) study. JAMA 274, 639-644.

Received 1 April 1998; revised 3 July 1998; accepted 7 July 1998. 